\title{
EXISTENCE OF PERIODIC SOLUTIONS FOR SEMILINEAR PARABOLIC EQUATIONS
}

\author{
NORIMICHI HIRANO \\ Department of Mathematics, Faculty of Engineering, \\ Yokohama National University, \\ Tokiwadai, Hodogaya-ku, Yokohama 156, Japan \\ E-mail: hirano@math.sci.ynu.ac.jp \\ NORIKO MIZOGUCHI \\ Department of Information Science, \\ Tokyo Institute of Technology \\ Oh-okayama, Meguro-ku, Tokyo 152, Japan
}

Abstract. In this paper, we are concerned with the semilinear parabolic equation

$$
\begin{cases}\frac{\partial u}{\partial t}-\Delta u=g(t, x, u) & \text { if }(t, x) \in \mathbf{R}_{+} \times \Omega \\ u=0 & \text { if }(t, x) \in \mathbf{R}_{+} \times \partial \Omega,\end{cases}
$$

where $\Omega \subset \mathbf{R}^{N}$ is a bounded domain with smooth boundary $\partial \Omega$ and $g: \mathbf{R}_{+} \times \bar{\Omega} \times \mathbf{R} \rightarrow \mathbf{R}$ is T-periodic with respect to the first variable. The existence and the multiplicity of T-periodic solutions for this problem are shown when $\frac{g(t, x, \xi)}{\xi}$ lies between two higher eigenvalues of $-\Delta$ in $\Omega$ with the Dirichlet boundary condition as $\xi \rightarrow \pm \infty$.

1. Introduction. Let $\Omega$ be a bounded domain in $\mathbf{R}^{N}$ with smooth boundary $\partial \Omega$ and $g \in C^{1, \alpha}\left(\mathbf{R}_{+} \times \bar{\Omega} \times \mathbf{R}\right)$ with $\alpha>0$ is T-periodic with respect to the first variable. In this paper, we are concerned with unstable T-periodic solutions for the semilinear parabolic equation

$$
(P) \quad\left\{\begin{aligned}
\frac{\partial u}{\partial t}-\Delta u & =g(t, x, u), & & (t, x) \in \mathbf{R}_{+} \times \Omega \\
u(t, x) & =0, & & (t, x) \in \mathbf{R}_{+} \times \partial \Omega .
\end{aligned}\right.
$$

1991 Mathematics Subject Classification: Primary 35K20; Secondary 35K55, 35B10.

The paper is in final form and no version of it will be published elsewhere. 
Many authors have studied the existence of periodic solutions not only for the problem (P) but also for a more general problem of the form

$$
(A P) \quad \frac{d u}{d t}+A u=F(t, u),
$$

where $A$ is an m-accretive operator (linear or nonlinear) on a Banach space $X$ and $F: \mathbf{R}_{+} \times X \rightarrow X$ is a continuous mapping which is T-periodic with respect to the first valiable. The existence and multiplicity of periodic solutions for problem $(\mathrm{P})$ is established by Amann [2] The abstract problem (AP) is studied in [7], [11] and [12].

For the existence of periodic solutions, it is usually assumed that the operator $A-F$ satisfies coercivity conditions. In the case of problem $(\mathrm{P})$, the operator $-\Delta-g(*)$ is coercive if

$$
\limsup _{|\xi| \rightarrow \infty} \sup \{|g(t, x, \xi) / \xi|:(t, x) \in[0, T] \times \Omega\}<\lambda_{1} .
$$

Here $\lambda_{1}$ is the first eigenvalue of the Laplacian on $\Omega$ with Dirichlet boundary condition.

Our purpose in this paper is to consider the existence and multiplicity of T-periodic solutions for $(\mathrm{P})$ when $\lim \sup _{|\xi| \rightarrow \infty} \frac{g(t, x, \xi)}{\xi}$ lies between two higher eigenvalues of the Laplacian on $\Omega$ with Dirichlet boundary condition. We also show the instability of Tperiodic solutions for $(\mathrm{P})$. For the stability and instability of periodic solutions for $(\mathrm{P})$, we refer to Alikakos, Hess and Matano [1], Hess [6], Hirano [9] and Hirsch [10].

2. Case of a general nonlinearity $g(t, x, \xi)$. Throughout the rest of this paper, we fix a positive number $T$. Let $|\cdot|$ and $\|\cdot\|$ be the norms of $L^{2}(\Omega)$ and $L^{2}\left(0, T ; L^{2}(\Omega)\right)$, respectively. The inner products of $L^{2}(\Omega)$ and $L^{2}\left(0, T ; L^{2}(\Omega)\right)$ are denoted by $\langle\cdot, \cdot\rangle$ and $\ll \cdot, \gg$, respectively. We call $u: \mathbf{R}_{+} \rightarrow H_{0}^{1}(\Omega)$ a T-periodic solution for the problem (P) provided that $u \in W^{1,2}\left(0, T ; L^{2}(\Omega)\right) \cap L^{2}\left(0, T ; H^{2}(\Omega)\right) \cap L^{2}\left(0, T ; H_{0}^{1}(\Omega)\right)$ satisfies

$$
\frac{\partial u}{\partial t}-\Delta u=g(t, x, u)
$$

in $L^{2}(\Omega)$ a.e. in $(0, T)$ and $u(t+T)=u(t)$ for all $t \in \mathbf{R}_{+}$. A T-periodic solution $u$ is said to be stable if for any $\epsilon>0$, there exists $\delta(\epsilon)>0$ such that for each $v_{0} \in L^{2}(\Omega)$ with $\left|v_{0}-u(0)\right|<\delta(\epsilon)$, it holds that $|v(t)-u(t)|<\epsilon$ for all $t>0$, where $v(t):(0, \infty) \rightarrow$ $H^{2}(\Omega) \cap H_{0}^{1}(\Omega)$ is the solution of the initial value problem

$$
(I) \quad\left\{\begin{aligned}
\frac{\partial v}{\partial t}-\Delta v & =g(t, x, v) & & \text { in }(0, \infty) \times \Omega \\
v & =0 & & \text { on }(0, \infty) \times \partial \Omega \\
v(0) & =v_{0} & & \text { in } \Omega .
\end{aligned}\right.
$$

A T-periodic solution $u$ is called unstable if $u$ is not stable.

Let $0<\lambda_{1}<\lambda_{2} \leq \lambda_{3} \cdots$ be the sequence of the eigenvalues of the boundary value problem

$$
\left\{\begin{aligned}
-\Delta u & =\lambda u & & \text { in } \Omega \\
u & =0 & & \text { on } \partial \Omega .
\end{aligned}\right.
$$

We denote by $\varphi_{i}$ an eigenfunction corresponding to $\lambda_{i}$. Throughout this paper, it is supposed that $g \in C^{1, \alpha}\left(\mathbf{R}_{+} \times \bar{\Omega} \times \mathbf{R}\right)$ with $\alpha>0$ is T-periodic with respect to the first 
variable. We assume the following conditions on $g$ :

i) There exists $M>0$ satisfying

$$
\lambda_{1} \leq \frac{\partial g}{\partial \xi}(t, x, \xi) \leq M \quad \text { for all }(t, x, \xi) \in \mathbf{R}_{+} \times \bar{\Omega} \times \mathbf{R}
$$

and

$$
\frac{\partial g}{\partial \xi}(t, x, 0)>\lambda_{1} \quad \text { for some }(t, x) \in \mathbf{R}_{+} \times \partial \Omega .
$$

ii) There are $m \geq 1$ and $\alpha>0$ such that

$$
\lambda_{m}+\alpha \leq \liminf _{\xi \rightarrow \pm \infty} \frac{g(t, x, \xi)}{\xi} \leq \limsup _{\xi \rightarrow \pm \infty} \frac{g(t, x, \xi)}{\xi} \leq \lambda_{m+1}-\alpha
$$

uniformly for $(t, x) \in \mathbf{R}_{+} \times \Omega$.

The purpose of this section is to prove the following results.

Theorem 1. Under the hypotheses i) and ii), the problem $(P)$ possesses an unstable T-periodic solution.

In case that $g(t, x, 0)=0$ for all $(t, x) \in \mathbf{R}_{+} \times \Omega, u \equiv 0$ is a T-periodic solution for $(\mathrm{P})$. Then $u \equiv 0$ may be unstable. We can prove the existence of a nontrivial unstable T-periodic solution for $(\mathrm{P})$ assuming the following condition :

iii) There are $2 \leq l \leq m$ and $\beta>0$ such that

$$
\lambda_{l-1}+\beta \leq \liminf _{\xi \rightarrow 0} \frac{g(t, x, \xi)}{\xi} \leq \limsup _{\xi \rightarrow 0} \frac{g(t, x, \xi)}{\xi}<\lambda_{1}-\beta
$$

uniformly for $(t, x) \in \mathbf{R}_{+} \times \Omega$.

THEOREM 2. Under the assumptions $i)-$ iii), if $m-l+1$ is an odd integer, then there exists a nontrivial unstable T-periodic solution for the problem $(P)$. Moreover if there exists a nontrivial T-periodic solution u for $(P)$ which is nondegenerate, i.e., 0 is not an eigenvalue of the problem

$$
(L) \quad\left\{\begin{aligned}
\frac{\partial u}{\partial t}-\Delta v-g^{\prime}(t, x, u) v & =\mu v & & \text { in } \mathbf{R}_{+} \times \Omega \\
v & =0 & & \text { on } \mathbf{R}_{+} \times \partial \Omega \\
v(T) & =v(0) & & \text { in } \Omega,
\end{aligned}\right.
$$

then the problem $(P)$ possesses at least two nontrivial unstable T-periodic solutions.

For simplicity, we write $H=L^{2}\left(0, T ; L^{2}(\Omega)\right)$ and $\frac{\partial g}{\partial \xi}(t, x, \xi)=g^{\prime}(t, x, \xi)$. Let

with domain

$$
L=\frac{\partial}{\partial t}-\Delta
$$

$$
D(L)=\left\{u \in W^{1,2}\left(0, T ; L^{2}(\Omega)\right) \cap L^{2}\left(0, T ; H^{2}(\Omega)\right) \cap L^{2}\left(0, T ; H_{0}^{1}(\Omega)\right): u(0)=u(T)\right\} .
$$

It is well known that there is a unique solution $u_{f}$ for $L u_{f}=f$ for any $f \in H$ and the operator $K$ defined by $K=L^{-1}$ is a compact mapping from $H$ into $H$. It is easy to see that $u$ is a T-periodic solution for (P) if and only if $u$ is a fixed point of $K \circ g$. 
LEMMA 1. Under the assumptions of Theorem 1, there is $R>0$ such that

$$
\operatorname{deg}\left(I-K \circ g, B_{R}(0), 0\right)=(-1)^{m},
$$

where deg means the Leray-Schauder degree and $B_{R}(u)$ is the closed ball in $H$ with radius $R$ centered at $u$.

Proof. Let $E_{1}$ and $E_{2}$ be the closed subspaces of $L^{2}(\Omega)$ spanned by $\left\{\varphi_{i}: i \geq m+1\right\}$ and $\left\{\varphi_{i}: 1 \leq i \leq m+1\right\}$, respectively. We denote by $P_{i}$ the projection from $L^{2}(\Omega)$ onto $E_{i}$ for $i=1,2$. Since $L^{2}\left(0, T ; E_{1}\right)$ and $L^{2}\left(0, T ; E_{2}\right)$ are orthogonal in $H$ and $H=$ $L^{2}\left(0, T ; E_{1}\right) \oplus L^{2}\left(0, T ; E_{2}\right), P_{i}$ is canonically extended to the projection $\tilde{P}_{i}$ from $H$ onto $L^{2}\left(0, T ; E_{i}\right)$ for $i=1,2$. From the assumption ii), we obtain $C_{1}, C_{2}>0$ such that

$$
\left\langle-\Delta v-g(t, x, v), P_{1} v-P_{2} v\right\rangle \geq C_{1}|v|^{2}-C_{2}
$$

for each $v \in H^{2}(\Omega) \cap H_{0}^{1}(\Omega)$ and $t \in \mathbf{R}_{+}$by the usual argument for semilinear elliptic equations with the Dirichlet boundary condition ( see [8] ). It follows that

$$
\ll L v-g(t, x, v), \tilde{P}_{1} v-\tilde{P}_{2} v \gg \geq C_{1}\|v\|^{2}-C_{2} T
$$

for all $v \in D(L)$. Therefore there exists $R>0$ satisfying

$$
\ll L v-g(t, x, v), \tilde{P}_{1} v-\tilde{P}_{2} v \gg>0
$$

for any $v \in D(L)$ with $\|v\| \geq R$. Take $\lambda_{m}<a<\lambda_{m+1}$. We consider a homotopy of compact mappings defined by $\{K(s g+(1-s) a I): 0 \leq s \leq 1\}$. For each $s \in[0,1]$ and $v \in D(L)$ with $\|v\|=R$, we get

$$
\ll L v-\{s g(t, x, v)+(1-s) a v\}, \tilde{P}_{1} v-\tilde{P}_{2} v \gg>0 .
$$

This shows that

$$
v-K(s g(t, x, v)+(1-s) a v) \neq 0
$$

for all $v \in H$ with $\|v\|=R$. By the homotopy invariance of the Leray-Schauder degree, we have

$$
\operatorname{deg}\left(I-K \circ g, B_{R}(0), 0\right)=\operatorname{deg}\left(I-a K, B_{R}(0), 0\right) .
$$

Now, let $\nu_{1}, \cdots, \nu_{n}$ be the eigenvalues of $a K$ with $\nu_{i}>1$ for $1 \leq i \leq n$ and $\psi_{i}$ be an eigenfunction corresponding to $\nu_{i}$ for $1 \leq i \leq n$. Then for $1 \leq i \leq n$ it holds that

$$
L \psi_{i}=\frac{a}{\nu_{i}} \psi_{i} \quad \text { for } 1 \leq i \leq n
$$

From $\nu_{i}>1$, it follows that $\frac{a}{\nu_{i}}=\lambda_{j}$ for some $j$ with $1 \leq j \leq m$. On the other hand, for each $j$ with $1 \leq j \leq m, \frac{a}{\lambda_{j}}$ is an eigenvalue of $a K$ with $\frac{a}{\lambda_{j}}>1$. This implies $n=m$. Consequently, we see

$$
\operatorname{deg}\left(I-a K, B_{R}(0), 0\right)=(-1)^{m} .
$$

This completes the proof.

Lemma 2. Under the hypotheses of Theorem 2, there exists $r$ with $0<r<R$ satisfying

$$
\operatorname{deg}\left(I-K \circ g, B_{r}(0), 0\right)=(-1)^{l-1} .
$$

Proof. Let $F_{1}$ and $F_{2}$ be the closed subspaces of $L^{2}(\Omega)$ spanned by $\left\{\varphi_{i}: i \geq l\right\}$ and $\left\{\varphi_{i}: 1 \leq i \leq l-1\right\}$, respectively. For $i=1,2$, we denote by $Q_{i}$ and $\tilde{Q}_{i}$ the projections 
from $L^{2}(\Omega)$ onto $F_{i}$ and from $H$ onto $L^{2}\left(0, T ; F_{i}\right)$, respectively. By the assumptions ii) and iii), there are $d, \rho>0$ such that

$$
\left\langle-\Delta v-g(t, x, v), Q_{1} v-Q_{2} v\right\rangle \geq \rho|v|^{2}
$$

for all $v \in H^{2}(\Omega) \cap H_{0}^{1}(\Omega)$ with $0<|v|<d$ and $t \in \mathbf{R}_{+}$( see [8] ). Take $\lambda_{l-1}<b<\lambda_{l}$. Then we can see that there exists $C_{1}>0$ such that for any $s \in[0,1]$, if $v \in D(L)$ satisfies

$$
L v-\{s g(t, x, v)+(1-s) b v\}=0
$$

then

$$
\sup _{t \in[0, T]}|v(t)| \leq C_{1}\|v\| .
$$

In fact, if $v$ is a solution of (2) for some $0 \leq s \leq 1$, then we multiply (2) by $v$ and integrate over $[s, t]$, where $|v(\tau)|$ attain its minimal at $s$. Then

$$
|v(t)| \leq s\|g\|\|v\|+(1-s) b\|v\|+\|v\|^{2} / T .
$$

for all $t \in[s, T]$.

It then follows from the periodicity of $v$ that the existence of $C_{1}$ satisfying the inequality above. Put $r=\frac{d}{C_{1}}$. Suppose that

$$
L v_{s}-\left\{s g\left(t, x, v_{s}\right)+(1-s) b v_{s}\right\}=0
$$

for some $s \in[0,1]$ and $v_{s} \in D(L)$ with $0<\left\|v_{s}\right\| \leq r$. Since

$$
\sup _{t \in[0, T]}\left|v_{s}(t)\right| \leq d
$$

it follows from (1) that

$$
\ll L v_{s}-\left\{s g\left(t, x, v_{s}\right)+(1-s) b v_{s}\right\}, \tilde{Q}_{1} v_{s}-\tilde{Q}_{2} v_{s} \gg>0 .
$$

This is a contradiction. Therefore we have

$$
v-K(s g(t, x, v)+(1-s) b v) \neq 0
$$

for each $v \in H$ with $0<\|v\| \leq r$. According to the homotopy invariance of the LeraySchauder degree, it follows that

$$
\operatorname{deg}\left(I-K \circ g, B_{r}(0), 0\right)=\operatorname{deg}\left(I-b K, B_{r}(0), 0\right) .
$$

By the same method as in the proof of Lemma 1, we obtain

$$
\operatorname{deg}\left(I-b K, B_{r}(0), 0\right)=(-1)^{l-1} .
$$

This completes the proof.

We next consider a sufficient condition for a T-periodic solution of the problem (P) to be unstable. Let $u$ be a T-periodic solution for $(\mathrm{P})$. Denote by $S(t, s)$ the evolution operator for the following problem

$$
\left\{\begin{aligned}
\frac{d v}{d t}-\Delta v & =g^{\prime}(t, x, u) v & & \text { in }(s, \infty) \times \Omega \\
v & =0 & & \text { on }(s, \infty) \times \partial \Omega \\
v(s) & =z & & \text { in } \Omega,
\end{aligned}\right.
$$


that is, $S(t, s) z=v(t)$. Then nonzero eigenvalues of $U(t)$ is independent of $t$ ( see [5] ). It is known that if the periodic map $U(t)=S(t+T, t)$ for the above problem satisfies

$$
\sigma(U(t)) \cap\{\mu:|\mu|>1\} \neq \emptyset,
$$

where $\sigma(A)$ means the set of eigenvalues of a linear operator $A$, then $u$ is unstable ( see Theorem 8.1.2 of [5]).

Putting $L_{u}=L+\left(M-g^{\prime}(t, x, u)\right)$ with domain $D(L)$, it was shown that $L_{u}$ has the real principal eigenvalue with an associated positive eigenfunction in Beltramo and Hess[3].

LEMma 3. Under the assumption $i)$, if $u$ is a T-periodic solution for $(P)$, then $u$ is unstable.

Pr o of. Suppose that $\sigma\left(L_{u}\right) \cap(-\infty, M)=\emptyset$. Let $\mu$ be the principal eigenvalue of $L_{u}$ and $\varphi_{\mu}$ be an eigenfunction corresponding to $\mu$. Then we have $\mu-M \geq 0, \varphi_{\mu}>0$ and

$$
L \varphi_{\mu}-g^{\prime}(t, x, u) \varphi_{\mu}=(\mu-M) \varphi_{\mu} .
$$

On the other hand, it holds that

$$
L \varphi_{1}=\lambda_{1} \varphi_{1}
$$

From (3) and (4), it follows that

$$
\begin{aligned}
& \int_{0}^{T} \int_{\Omega}\left(g^{\prime}(t, x, u)+\mu-M-\lambda_{1}\right) \varphi_{\mu} \varphi_{1} d x d t \\
& =\int_{0}^{T} \int_{\Omega}\left\{\left(\varphi_{\mu}\right)_{t} \varphi_{1}-\left(\Delta \varphi_{\mu}\right) \varphi_{1}-\left(-\Delta \varphi_{1}\right) \varphi_{\mu}\right\} d x d t \\
& =0 .
\end{aligned}
$$

By the assumption i), this is a contradiction. This implies $\sigma\left(L_{u}\right) \cap(-\infty, M) \neq \emptyset$. Let $\mu=M+\gamma$ be an eigenvalue of $L_{u}$ with $\gamma<0$ and $\varphi_{\gamma}$ be an eigenfunction corresponding to $M+\gamma$. Then it holds that

$$
\frac{d \varphi_{\gamma}}{d t}-\Delta \varphi_{\gamma}-g^{\prime}(t, x, u) \varphi_{\gamma}=\gamma \varphi_{\gamma}
$$

and hence

$$
\frac{d\left(e^{-\gamma t} \varphi_{\gamma}\right)}{d t}-\Delta\left(e^{-\gamma t} \varphi_{\gamma}\right)-g^{\prime}(t, x, u)\left(e^{-\gamma t} \varphi_{\gamma}\right)=0
$$

This implies that $e^{-\gamma t} \varphi_{\gamma}$ is a solution of the initial value problem (LI) with $z=\varphi_{\gamma}(0)$. Then we get $U(0) \varphi_{\gamma}(0)=e^{-\gamma T} \varphi_{\gamma}(0)$, that is, $U(0)$ has an eigenvalue $e^{-\gamma T}>1$. Therefore $u$ is unstable. This completes the proof.

We can prove Theorems 1,2 using Lemmas 1-3.

Proof of Theorem 1. By Lemma 1, we obtain a T-periodic solution $u$ for the problem $(\mathrm{P})$. Lemma 3 shows that this solution $u$ is unstable.

Proof of Theorem 2. From Lemmas 1 and 2, it follows that

$$
\operatorname{deg}\left(I-K \circ g, B_{R}(0) \backslash B_{r}(0), 0\right) \neq 0
$$

since $m-l+1$ is an odd integer. Therefore there exists a nontrivial T-periodic solution $u$ for $(\mathrm{P})$. By Lemma 3, this $u$ is an unstable T-periodic solution of $(\mathrm{P})$. Next assume the 
existence of nondegenerate nontrivial T-periodic solution $u$ for $(\mathrm{P})$. Since the problem (L) do not have 0 as an eigenvalue, $I-K \circ g^{\prime}(u)$ is invertible. Let $k$ be the sum of the algebraic multipliers of the eigenvalues of $(\mathrm{L})$ greater than 1 . Then we have

$$
\operatorname{deg}\left(I-K \circ g, B_{\varepsilon}(u), 0\right)=(-1)^{k},
$$

for sufficiently small $\varepsilon>0$. Therefore it holds from Lemmas 1 and 2 that

$$
\operatorname{deg}\left(I-K \circ g, B_{R}(0) \backslash\left(B_{r}(0) \cup B_{\varepsilon}(u)\right), 0\right) \neq 0 .
$$

This implies the existence of another nontrivial T-periodic solution of $(\mathrm{P})$.

Remark 1. Under the hypotheses of Theorem $2, u \equiv 0$ is an unstable T-periodic solution for $(\mathrm{P})$ by Lemma 3 .

3. Case of $g(t, x, \xi)=f(\xi)+h(t, x)$. In the present section, we consider the special case that $g(t, x, \xi)=f(\xi)+h(t, x)$ for $(t, x, \xi) \in \mathbf{R}_{+} \times \bar{\Omega} \times \mathbf{R}$, where $f \in C^{1, \alpha}(\mathbf{R})$ and $h \in C^{1, \alpha}\left(\mathbf{R}_{+} \times \bar{\Omega}\right)$ which is T-periodic with respect to the first variable.

THEOREM 3. Under the assumptions $i)$, ii), if $\lambda_{l-1}<f^{\prime}(0)<\lambda_{l}$ for some $l \in \mathbf{N}$ with $2 \leq l \leq m$ and $m-l+1$ is odd, then the problem $(P)$ with $g(t, x, \xi)=f(\xi)+h(t, x)$ has at least two unstable T-periodic solutions for $h$ with $\|h\|$ sufficiently small. Moreover if all T-periodic solutions for $(P)$ are nondegenerate, then there exist at least three unstable T-periodic solutions for $(P)$.

P r o of. By the same argument as in the proof of Lemma 2, there are positive numbers $\delta, \omega$ satisfying that

$$
\left\langle L v-f(v), Q_{1} v-Q_{2} v\right\rangle \geq \omega|v|^{2}
$$

for all $v \in H^{2}(\Omega) \cap H_{0}^{1}(\Omega)$ with $0<|v| \leq \delta$. Take $\lambda_{l-1}<b<\lambda_{l}$. By the same argument as in the proof of Lemma 2, we obtain $C_{2}>0$ such that for any $s \in[0,1]$, if $v \in D(L)$ satisfies

$$
L v-\{s g(t, x, v)+(1-s) b v\}=0,
$$

then

$$
\sup _{t \in[0, T]}|v(t)| \leq C_{2}(\|v\|+\|h\|) .
$$

Let $r<\frac{\delta}{2 C_{2}}$ and $\|h\|<\min \left\{\frac{\delta}{2 C_{2}}, \omega r\right\}$. Suppose that

$$
L v_{s}-\left\{s g\left(t, x, v_{s}\right)+(1-s) b v_{s}\right\}=0
$$

for some $s \in[0,1]$ and $v_{s} \in D(L)$ with $\left\|v_{s}\right\|=r$. Since

$$
\sup _{t \in[0, T]}\left|v_{s}(t)\right| \leq \delta
$$

it follows from (2) that

$$
\ll L v_{s}-\left\{s g\left(t, x, v_{s}\right)+(1-s) b v_{s}\right\}, \tilde{Q}_{1} v_{s}-\tilde{Q}_{2} v_{s} \gg>0 .
$$

This is a contradiction. Therefore we get

$$
v-K\left\{s g\left(t, x, v_{s}\right)+(1-s) b v\right\} \neq 0
$$


for all $v \in H$ with $\|v\|=r$. By the same method as in the proof of Lemma 2, it holds that

$$
\operatorname{deg}\left(I-K \circ g, B_{r}(0), 0\right)=(-1)^{l-1} .
$$

In order to show the rest of the proof, it is sufficient to take the same process as in the proof of Theorem 2 .

We next give a sharper result than the above theorem. A solution $w$ of the semilinear elliptic problem

$$
(S) \quad\left\{\begin{array}{rlrl}
-\Delta w & =f(w) & \text { in } \Omega \\
w & =0 & & \text { on } \partial \Omega
\end{array}\right.
$$

is said to be nondegenerate if 0 is not an eigenvalue of the linearized problem of $(\mathrm{S})$

$$
\left\{\begin{aligned}
-\Delta v-f^{\prime}(w) v & =\lambda v & & \text { in } \Omega \\
w & =0 & & \text { on } \partial \Omega .
\end{aligned}\right.
$$

The stability and instability of solutions for $(\mathrm{S})$ are defined as same as those of T-periodic solutions for $(\mathrm{P})$.

THEOREM 4. Under the hypotheses of Theorem 3, if $l=m$ and $f^{\prime}$ is strictly increasing on $[0,+\infty)$ and strictly decreasing on $(-\infty, 0)$, then the problem $(P)$ with $g(t, x, \xi)=$ $f(\xi)+h(t, x)$ possesses at least three unstable T-periodic solutions for $h$ with $\|h\|>0$ sufficiently small.

R e m a r k 2. From the proof of Theorem 4, we can see that if $\|h\|$ is sufficiently small, then there are three unstable solutions $u_{1}, u_{2}, u_{3}$ and they lie in small neighborhoods in $L^{2}\left(0, T ; L^{2}(\Omega)\right)$ of unstable solutions $w_{1}, w_{2}, 0$ for $(\mathrm{S})$, respectively.

We need the following two lemmas.

LEMMA 4. Under the assumptions of Theorem 4 , if $w$ is a solution for $(S)$, then there are $\delta_{1}, \rho_{1}>0$ such that for $\delta \leq \delta_{1}$ and $0<\|h\| \leq \rho_{1} \delta$,

$$
\operatorname{deg}\left(I-K \circ g, B_{\delta}(w), 0\right\}=(-1)^{n},
$$

where $n$ is the sum of the multiplicities of the eigenvalues of $K \circ f^{\prime}(w)$ greater than 1 .

Proof. Let $X_{1}$ and $X_{2}$ be closed subspaces of $L^{2}(\Omega)$ spanned by eigenfunctions corresponding to the eigenvalues of (SL) greater and less than 0 , respectively. Then $X_{1}$ and $X_{2}$ are orthogonal. Denote by $Q_{i}$ and $\tilde{Q}_{i}$ the projections of $L^{2}(\Omega)$ onto $X_{i}$ and the canonically extended projection of $Q_{i}$ on $H$ onto $L^{2}\left(0, T ; X_{i}\right)$ for $i=1,2$, respectively. It is easy to see the existence of some positive number $\gamma$ satisfying

$$
\int_{\Omega}\left(-\Delta v-f^{\prime}(w) v\right)\left(Q_{1} v-Q_{2} v\right) d x \geq \gamma|v|^{2}
$$

for all $v \in H^{2}(\Omega) \cap H_{0}^{1}(\Omega)$. Since $f: H \rightarrow H$ is of class $C^{1}$, we get

$$
f(u)=f(w)+f^{\prime}(u-w)+\phi(u-w)
$$

for $u \in H$, where $\phi \in o(\|v\|)$ as $\|v\| \rightarrow 0$. It follows that

$$
L u-g(t, x, u)=L(u-w)-f^{\prime}(w)(u-w)-\phi(u-w)-h .
$$


Therefore for $s \in[0,1]$ and $u \in D(L)$, we have

$$
\begin{gathered}
\ll s\{L u-g(t, x, u)\}+(1-s)\left\{L(u-w)-f^{\prime}(w)(u-w)\right\}, \\
\tilde{Q}_{1}(u-w)-\tilde{Q}_{2}(u-w) \gg \\
=\ll L(u-w)-f^{\prime}(w)(u-w) s \phi(u-w)-s h, \\
\tilde{Q}_{1}(u-w)-\tilde{Q}_{2}(u-w) \gg \\
=\int_{0}^{T} \int_{\Omega}\left\{u_{t}-\Delta(u-w)-f^{\prime}(w)(u-w)-s \phi(u-w)-s h\right\} \\
\left\{Q_{1}(u-w)-Q_{2}(u-w)\right\} d x d t \\
=\int_{0}^{T} \int_{\Omega}\left\{-\Delta(u-w)-f^{\prime}(w)(u-w)-s \phi(u-w)-s h\right\} \\
\left\{Q_{1}(u-w)-Q_{2}(u-w)\right\} d x d t \\
\geq \gamma\|u-w\|^{2}-(\|\phi(u-w)\|+\|h\|)\|u-w\| .
\end{gathered}
$$

By $\phi(v) \in o(\|v\|)$, for $0<\varepsilon<\gamma$ there is $\delta_{\varepsilon}>0$ such that $\|\phi(v)\| \leq \varepsilon\|v\|$ if $\|v\| \leq \delta_{\varepsilon}$. Taking $\delta_{1}<\delta_{\varepsilon}$ and $\rho_{1}=\gamma-\varepsilon$, if $\delta \leq \delta_{1}$ and $\|h\| \leq \rho_{1} \delta$, then it holds that

$$
\begin{aligned}
\ll s\{L u-g(t, x, u)\}+(1-s)\left\{L(u-w)-f^{\prime}(w)(u-w)\right\}, \\
\tilde{Q}_{1}(u-w)-\tilde{Q}_{2}(u-w) \gg>0
\end{aligned}
$$

for $s \in[0,1]$ and $u \in \partial B_{\delta}(w)$. This shows that

$$
s\{u-K \circ g(t, x, u)\}+(1-s)\left\{u-w-K \circ f^{\prime}(w)(u-w)\right\} \neq 0
$$

for $s \in[0,1]$ and $u \in \partial B_{\delta}(w)$. According to the homotopy invariance of the LeraySchauder degree, it follows that

$$
\operatorname{deg}\left(I-K \circ g, B_{\delta}(w), 0\right)=\operatorname{deg}\left(I-K \circ f^{\prime}(w), B_{\delta}(0), 0\right) .
$$

Suppose that

$$
K \circ f^{\prime}(w) v=v
$$

i.e.,

$$
v_{t}-\Delta v-f^{\prime}(w) v=0
$$

for some $v \neq 0$. Multiplying this equality by $v_{t}$ and integrating on $(0, T) \times \Omega$, we obtain $v_{t} \equiv 0$ and hence

$$
-\Delta v=f^{\prime}(w) v,
$$

which contradicts that $w$ is nondegenerate. This implies that 1 is not an eigenvalue of $K \circ f^{\prime}(w)$. Consequently, we see

$$
\operatorname{deg}\left(I-K \circ f^{\prime}(w), B_{\delta}(0), 0\right)=(-1)^{n},
$$

where $n$ is the sum of the multiplicities of the eigenvalues of $K \circ f^{\prime}(w)$ greater than 1 . This completes the proof.

We investigate a relation for stability and instability between a solution for $(\mathrm{S})$ and a T-periodic solution for $(\mathrm{P})$. For a solution $w$ of $(\mathrm{S})$ and a T-periodic solution $u$ of $(\mathrm{P})$, 
denote by $\lambda_{w}$ and $\mu_{u}$ the first eigenvalue of (SL) and a real principal eigenvalue of (L), respectively.

Lemma 5. Let $w \in C^{2}(\bar{\Omega})$ be a solution of the problem $(S)$ which is nondegenerate. Then there exist $\delta_{2}, \rho_{2}>0$ such that if $u \in B_{\delta_{2}}(w)$ is a T-periodic solution for $(P)$ with $g(t, x, \xi)=f(\xi)+h(t, x)$ with $\|h\| \leq \rho_{2}$, then $u$ is nondegenerate and the sign of $\mu_{u}$ coincides with that of $\lambda_{w}$.

Proof. Suppose that $u$ is a T-periodic solutions for $(\mathrm{P})$ and $w$ is a solution for (S). Let $\varphi$ and $\psi$ be positive eigenfunctions corresponding to $\lambda_{w}$ and $\mu_{u}$, respectively. Then it holds that

$$
\int_{0}^{T} \int_{\Omega}\left\{f^{\prime}(u)-f^{\prime}(w)-\lambda_{w}+\mu_{u}\right\} \varphi \psi d x d t=0 .
$$

By $f \in C^{1, \alpha}(\mathbf{R})$, there is $C_{1}>0$ satisfying that

$$
\left|f^{\prime}\left(\xi_{1}\right)-f^{\prime}\left(\xi_{2}\right)\right| \leq C_{1}\left|\xi_{1}-\xi_{2}\right|^{\alpha}
$$

for $\xi_{1}, \xi_{2} \in \mathbf{R}$. Since $u$ is a T-periodic solution for $(\mathrm{P})$ and $w$ is a solution for $(\mathrm{S})$, it follows that

$$
\frac{\partial(u-w)}{\partial t}-\Delta(u-w)-\{f(u)-f(w)\}-h=0 .
$$

On the other hand we have by the same argument as in the proof of Lemma 2, there are $\delta_{2}, \rho_{2}>0$ such that

$$
\sup _{(t, x) \in[0, T] \times \bar{\Omega}}|u(t, x)-w(x)|<\left(\frac{\left|\lambda_{w}\right|}{C_{1}}\right)^{1 / \alpha}
$$

if $\|h\| \leq \rho_{2}$ and $u \in B_{\delta_{2}}(w)$ is any T-periodic solution for $(\mathrm{P})$ with $g(t, x, \xi)=f(\xi)+$ $h(t, x)$ since $f$ is Lipschitz continuous. Let $\|h\| \leq \rho_{2}$ and $u \in B_{\delta_{2}}(w)$ be a solution for (P). In the case of $\lambda_{w}<0$, assuming that $\mu_{u} \geq 0$, we have by (7) and (8),

$$
f^{\prime}(u)-f^{\prime}(w)-\lambda_{w}+\mu_{u}>0,
$$

which contradicts (6). This implies that $\mu_{u}<0$. By the same argument as the above, we can prove the case of $\lambda_{w}>0$. This completes the proof.

Proof of Theorem 4. Under the hypotheses of Theorem 4, there exist at least two nontrivial solution $w_{1}$ and $w_{2}$ in $C^{2}(\bar{\Omega})$ for $(\mathrm{S})$ which are nondegenerate and unstable (see $[4])$. It is immediate that 0 is nondegenerate unstable solution for (S). Choosing positive numbers $\delta$ and $\rho$ sufficiently small, by lemmas 4 and 5 , there are at least three unstable T-periodic solutions $u_{1}, u_{2}, u_{3}$ for $(\mathrm{P})$ with $g(t, x, \xi)=f(\xi)+h(t, x)$ and $0<\|h\| \leq \rho$ such that $u_{i} \in B_{\delta}\left(w_{i}\right)$ for $i=1,2$ and $u_{3} \in B_{\delta}(0)$.

Both stable T-periodic solutions and unstable ones exist in the following cases.

TheOREM 5. Suppose that

$$
f^{\prime}(0)<\lambda_{1}<\liminf _{|\xi| \rightarrow \infty} \frac{g(\xi)}{\xi} \leq \limsup _{|\xi| \rightarrow \infty} \frac{g(\xi)}{\xi}<\lambda_{2}
$$


and $f^{\prime}$ is strictly increasing on $[0, \infty)$ and strictly decreasing on $(0, \infty)$. Then the problem $(P)$ with $g(t, x, \xi)=f(\xi)+h(t, x)$ has at least one stable T-periodic solution and two unstable T-periodic solutions if $\|h\|>0$ is sufficiently small.

P r o of. By [4], there are at least two nontrivial solutions of $(\mathrm{S})$ which are nondegenerate and unstable. Obviously, 0 is a stable solution for (S). Using Lemmas 4 and 5, we can obtain the consequence of this theorem.

\section{References}

[1] N. D. Alikakos, P. Hess and H. Matano, Discrete order preserving semigroups and stability for periodic parabolic differential equaitons, J, Diff. Eq. 82 (1989), 322-341.

[2] H. Amann, Periodic solutions for semi-linear parabolic equations, in "Nonlinear Analysis: A Collection of Papers in Honor of Erich Rothe", Academic Press, New York, 1978, $1-29$.

[3] A. Beltramo and P. Hess, On the principal eigenvalue of a periodic-parabolic operator, Comm. Part. Diff. Eq. 9 (1984), 919-941.

[4] A. Castro and A. Lazer, Critical point theory and the number of solutions of a Dirichlet problem, Ann. Math. Pure Appl. 70 (1979), 113-137.

[5] D. Henry, Geometric theory of semilinear parabolic equaitons, Lecture Notes in Math. 840, Springer-Verlag, New York, 1981.

[6] P. Hess, On positive solutions of semilinear periodic-parabolic problems in infinite -dimensional systems, ed. Kappel-Schappacher, Lecture Notes in Math. 1076 (1984), 101-114.

[7] N. Hirano, Existence of multiple periodic solutions for a semilinear evolution equations, Proc. Amer. Math. Soc. 106 (1989), 107-114.

[8] _ Existence of nontrivial solutions of semilinear elliptic equaitons, Nonlinear Anal. 13 (1989), 695-705.

[9] Existence of unstable periodic solutions for semilinear parabolic equations, to appear in Nonlinear Analysis.

[10] M. W. Hirsch, Differential equations and convergence almost everywhere in strongly monotone semiflows, Contemporary Math. 17 (1983), 267-285.

[11] J. Pr üss, Periodic solutions of semilinear evolution equations, Nonlinear Anal. 3 (1979), 601-612.

[12] I. I. Vrabie, Periodic solutions for nonlinear evolution equations in a Banach space, Proc. Amer. Math. Soc. 109 (1990), 653-661. 